\title{
MONITORING AND ASSESSMENT OF ACOUSTIC EMISSION SIGNATURES DURING FATIGUE MECHANISM OF API5LX70 GAS PIPELINE STEEL
}

\author{
M.F.M. Yusof ${ }^{1,2}$, N. Jamaludin ${ }^{1}$, S. Abdullah ${ }^{1}$, Z.H. Hanafi ${ }^{1}$, M.S.M. Zain ${ }^{1}$ \\ ${ }^{1}$ Department of Mechanical and Materials Engineering \\ Faculty of Engineering and Built Environment, Universiti Kebangsaan Malaysia, \\ 43600 UKM, Bangi, Selangor, Malaysia. \\ ${ }^{2}$ Faculty of Mechanical Engineering, Universiti Malaysia Pahang, \\ 26600 UMP, Pekan, Pahang, Malaysia; \\ Phone: +6019-7750510, Fax: +609-4242202 \\ E-mail: fadhlanyusof@gmail.com
}

\begin{abstract}
The detection of early fatigue phenomena in a gas pipeline is crucial in order to avoid catastrophic consequences. Therefore, appropriate inspection is needed to assess fatigue phenomena in a gas pipeline system. Acoustic emission (AE) technology is expected to be suitable in this regard. This paper presents the monitoring and assessment of AE signatures of the fatigue mechanism of gas pipeline material, API 5L X70 steel. The stress amplitude of $65 \%, 60 \%, 58 \%$ and $53 \%$ of the ultimate tensile strength were done in order to observe AE activity during the fatigue mechanism. Field measurements were also done by investigating $\mathrm{AE}$ signatures of an operational gas pipeline for comparison purposes. Based on the correlation of the $\mathrm{AE}$ signatures and fatigue mechanism, it was found that the $\mathrm{AE}$ activities generated during the fatigue mechanism were divided into three different stages. Analysis of the AE features and statistical parameters have shown that the kurtosis values of the time domain AE signatures from the third stage of the fatigue mechanism were different from the field measurements. This results show that the application of the kurtosis was expected to be able to detect the time domain $\mathrm{AE}$ signatures from the crack stage.
\end{abstract}

Keywords: Acoustic emission, fatigue, API5L X70 steel, statistical parameter.

\section{INTRODUCTION}

Fatigue is a common failure mechanism in engineering structures including gas pipelines. Even though historical evidence does not suggest that fatigue failure is a major problem in gas pipelines; it cannot be ignored because it can happen rapidly, without any indication and cause a catastrophic failure (Muhlbauer, 2004). Conventionally, inspection techniques of in-operation gas pipelines only gives information about noticeable cracks. However, it is not essential to detect noticeable cracks because at that stage, gas pipelines would already be considered failed. Thus, a technique for monitoring and early detection of fatigue is crucial. Inclusion and pores in some materials can influence the crack initiation mechanism (Chan, 2009). Both size and spacing of inclusions and pores affects localised strain and crack incubation life. Generally, a bigger size and smaller spacing of inclusions and pores increases the amount of local plastic strain and shortens the crack incubation life. In materials with fewer (approximately zero) numbers of inclusion and pores, cracks tend to initiate from the persistent slip band (PSB). The extension of intrusion and extrusion cause slip plane 
cracking to extend to a few grain diameters, which then changes to the continuum mechanism of crack propagation (Janssen, Zuidema, \& Wanhill, 2004). During crack growth, the mechanism of microplastic deformation at the crack tip and crack closure is involved, which has captured the attention for scientific study.

The acoustic emission (AE) technique is suitable because it can detect micro phenomena in materials. AE is defined as the radiation of elastic waves produced by a localised source, due to the dynamic local arrangement of the internal structure of a test material (Baranov, Kudryavtsev, Sarychev, \& Shcavelin, 2007). The source of AE can be classified as microscale or macroscale. A microscale source refers to micromechanisms, such as dislocation motion, slip formation, micro crack initiation, etc. (Kalyanasundaram, Mudhophadyay, \& SubraRao, 2007). Huang, Jiang, Liaw, Brooks, Seelay, and Klarstrom (1998) summarised that AE activities generated during fatigue of materials is caused by various mechanisms, which can be divided into three stages. The first stage shows high AE activity due to the dislocation movement and cyclic softening and hardening. These phenomena occur during the first few cycles before the cyclic curve becomes stabilised. The second stage corresponds to the crack incubation stage, where steady-state dislocation happens and results in the formation of micro voids and micro cracks. This phenomenon leads to a period when only small AE activity occurs. Ai, Liu, Chen, He, and Wang (2010) found that a few burst-type AE signals appear at this stage due to the micro crack formation. During the last stage, crack start to grow and propagate and $\mathrm{AE}$ activities reactivate. Much of the $\mathrm{AE}$ generated at this stage is because of micro crack coalescence, fractures along grain boundaries, crack tip plastic deformation and also crack closure. The crack initiation phenomenon can be detected by a rapid increase of $\mathrm{AE}$ activity at positive peak stress during cyclic loading of Incoloy 901 (Berkovits \& Fang, 1995). It was followed by AE activity with clear boundaries around zero stresses, which correspond to crack closure phenomena. After the crack propagates, an increase in crack length might affect the crack closure phenomena. Therefore, Chang, Han, Wang, and Ke (2009) undertook a study into the effect of crack length on the crack closure phenomena in aluminium alloy LY12CZ, using AE monitoring. Recognition of AE behaviour during crack closure was taken as a good guideline by Roberts and Talebzadeh (2003) in order to study the growth of cracks.

Fatigue crack mechanisms can be examined by extracting AE features from the generated AE signatures. AE features, such as hits (amount of AE activity), count and count rates have been successfully correlated with crack closure phenomena, micro phenomena during fatigue mechanism and prediction of crack growth and life. However, there is a lack of analyses of the statistical parameters of the time domain $\mathrm{AE}$ signatures generated during the fatigue crack mechanism. In the case of fatigue and vibration analysis, statistical parameters are frequently used to characterise and classify random signals (Nuawi, Abdullah, Abdullah, Haris, \& Arifin, 2009). Elangovan, BabuDevasenapati, Sakhtivel, and Ramachandran (2011) summarised that the kurtosis of time domain vibration signals is a good measurement for tool condition monitoring. This paper presents the monitoring and assessment of AE time domain signatures during the fatigue mechanism of a gas pipeline material, API 5L X70 steel, in a laboratory test and normal field operation. 


\section{METHODOLOGY}

\section{Experimental Setup}

The fatigue test specimens were prepared by cutting the test coupon of API 5L X70 steel in the longitudinal direction. It was milled into a plate and wire cut to dimensions according to the ASTM E466-07 standard. Figure 1 shows the data acquisition setup in the laboratory. The wide band piezoelectric sensor was mounted on the surface of the fatigue specimen using vacuum grease and calibrated by pencil lead break testing (ASTM E976-05). The threshold level of $44.1 \mathrm{~dB}$ was set once the background noise level was detected. Recorded AE signals were amplified by a preamplifier with $34 \mathrm{~dB}$ gain and analysed using an AMSY-5 from Vallen Systeme. Captured AE signals were filtered within a frequency range from $25-850 \mathrm{kHz}$ with a sampling rate of $5 \mathrm{MHz}$. The fully reverse fatigue test $(R=-1)$ with frequency of $5 \mathrm{~Hz}$ and stress amplitude of $65 \%$, $60 \%, 58 \%$ and $53 \%$ of its ultimate tensile strength were done based on ATSM E466-07. The cyclic stress amplitudes were varied in order to observe the pattern of AE activity. The stress ratio $R$, of -1 was selected in order to observe the behaviour of the $\mathrm{AE}$ spectrum generated from both tension and compression modes.

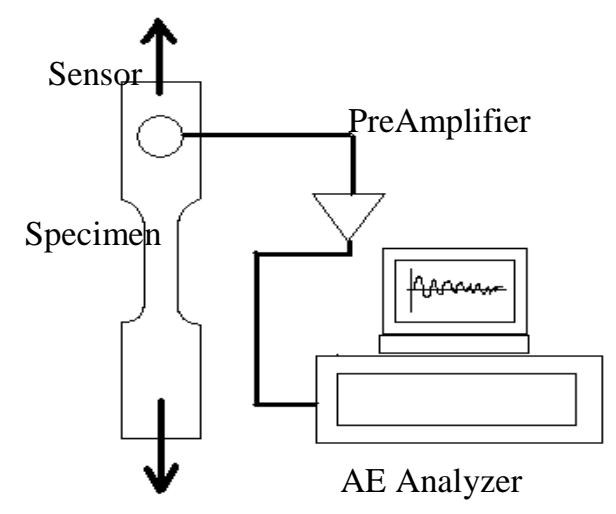

Figure 1. Data Acquisition setup in the laboratory.

\section{Field Measurement Setup}

It was obvious to capture AE signatures at the junction of a gas pipeline in the field. The measurement was done by mounting the wide band piezoelectric sensor on the surface of the pipeline using vacuum grease. The sensor mounting was calibrated by pencil lead break testing according to the ASTM E976-05 standard. All controlled parameters in the $\mathrm{AE}$ acquisition setup were similar to those of the laboratory test. However, in the field measurement, lower background noise was detected. Therefore, the threshold setting was decreased to $40 \mathrm{~dB}$. Figure 2 shows the field measurement setup.
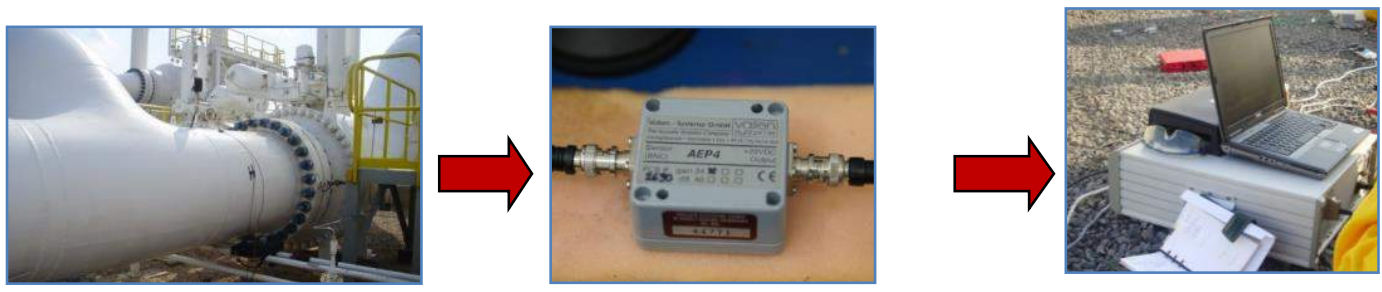

Figure 2. Field measurement setup. 


\section{Feature Extraction}

In this study, the correlation between the $\mathrm{AE}$ activity and fatigue mechanism were plotted in a 3D histogram. AE signatures were grouped based on those stages for the purpose of feature analysis. The time domain AE signatures from the laboratory fatigue test were divided into seven groups based on different stages of crack mechanism. The grouped time domain $\mathrm{AE}$ signatures from the fatigue mechanism were combined with the time domain $\mathrm{AE}$ signatures from the in-operation gas pipeline for feature extraction analysis. Figure 3 shows how AE features were extracted from the time-domain signatures. AE energy is the area under the graph within the duration of time domain AE signatures. Among all the AE features, only two were selected for analysis: the AE maximum amplitude and AE energy.

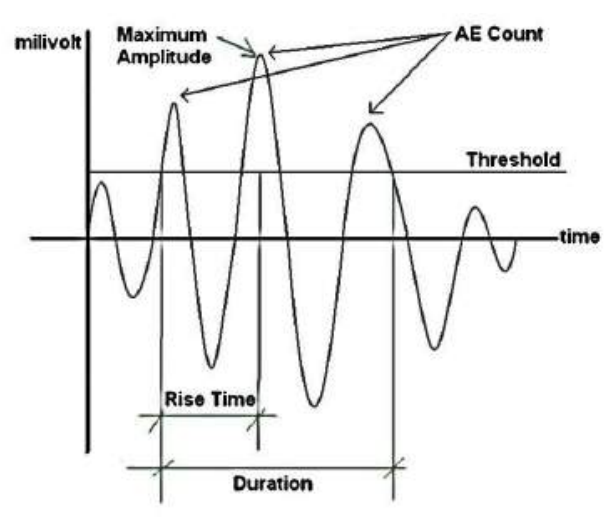

Figure 3. Time domain and frequency domain AE signatures.

The AE maximum amplitude was chosen for study because it can be related to the intensity of the source. It was expected that AE maximum amplitude would give a different value for different fatigue mechanisms. The energy of the AE signal has an advantage compared with other features because it can be related directly with the physical parameters of the AE source. In this study, AE energy will be presented in electrical units (eu). Therefore, the area under the graph of the AE time domain signatures in Eq. (1) will be converted into eu by using Eq. (2). It was explained that the threshold levels of the field measurement were different from those of the laboratory fatigue tests. Thus, the AE energy value from the field measurement signals will be calculated based on the laboratory threshold to avoid errors from the analysed data.

$$
\begin{aligned}
& \mathrm{E}=\frac{1}{R} \int_{0}^{\infty} \mathrm{V}(\mathrm{t})^{2} \cdot \mathrm{dt} \\
& 1 e u=10^{-14} V^{2}
\end{aligned}
$$

The most commonly used statistical parameters are mean, standard deviation, root mean square (rms), skewness, kurtosis and crest factor (Nuawi et al., 2009). However, only the standard deviation, kurtosis and skewness were selected for this study. The definition and formula of those statistical parameters are as follows:

(a) Standard deviation - This is defined as the measurement of data spreading about the mean value. This is also defined as a measure of the power content of the 
vibration signal (Elangovan et al., 2011). The standard deviation is expressed as Eq. (3).

$$
\text { Standard Deviation }=\left(\frac{1}{n-1} \sum_{\mathrm{j}=1}^{\mathrm{n}}\left(\mathrm{x}_{\mathrm{j}}-\overline{\mathrm{x}}\right)^{2}\right)^{\frac{1}{2}}
$$

(b) Kurtosis - Kurtosis is a measure of the peakedness of the data. It is used for detection of fault symptoms because it is highly sensitive to the high-amplitude event. Thus, kurtosis is sensitive in detecting fault phenomena, which are referred to as micro phenomena during the fatigue mechanism.

$$
\text { Kurtosis }=\frac{1}{n(\text { Standard Deviation })^{4}} \sum_{\mathrm{j}=1}^{\mathrm{n}}\left(\mathrm{x}_{\mathrm{j}}-\overline{\mathrm{x}}\right)^{4}
$$

(c) Skewness -It is the measure of symmetry or the data distribution, as expressed in Eq. (5).

$$
\text { Skewness }=\frac{1}{n(\text { StandardDeviation })^{3}} \sum_{\mathrm{j}=1}^{n}\left(\mathrm{x}_{\mathrm{j}}-\overline{\mathrm{x}}\right)^{3}
$$

The value of $n$ in Eqs (3)-(5) corresponds to the number of samples in single signatures. The time domain AE signatures were cut to avoid the effect of unwanted signatures in analysis. Thus, the value of $n$ in each signal in this study was 2500 .

\section{RESULTS AND DISCUSSION}

\section{AE Signatures and Fatigue Mechanism}

The correlation between AE activity and fatigue mechanism is presented in this section. Figure 4 shows the AE activity during the fatigue test with stress amplitude of $65 \%$ of the ultimate tensile strength. It can be clearly seen that the number of captured AE signatures were high during the first few cycles. It is found that this phenomenon occurs until the 25th cycle. From the 25th cycle to the 200th cycle, the AE activity is not as active; only a small number of $\mathrm{AE}$ activities were captured within this range. $\mathrm{AE}$ activity becomes more active again beyond the 200th cycle and the number of AE activities is found to increase rapidly starting from the 1920th cycle. However, most of the activity after the 1920th cycle is captured at positive peak stress amplitude. A similar trend is shown by AE activity during the fatigue test with stress amplitude of $60 \%$ of ultimate tensile strength, displayed in Figure 5. It is found that AE activities are active at the first few cycles. This phenomenon can be observed starting from the first cycles until the 20th cycle. After the 20th cycle, AE activity was not as active and only a small number of $\mathrm{AE}$ activities were captured. This phenomenon continued until the 100th cycle, when AE activity started to become active again. After 3500 cycles, AE activities become more active with a high number of AE activities per cycle. During the last stage before the experiment finished, high numbers of AE activities were also found at the positive peak stress amplitude. 


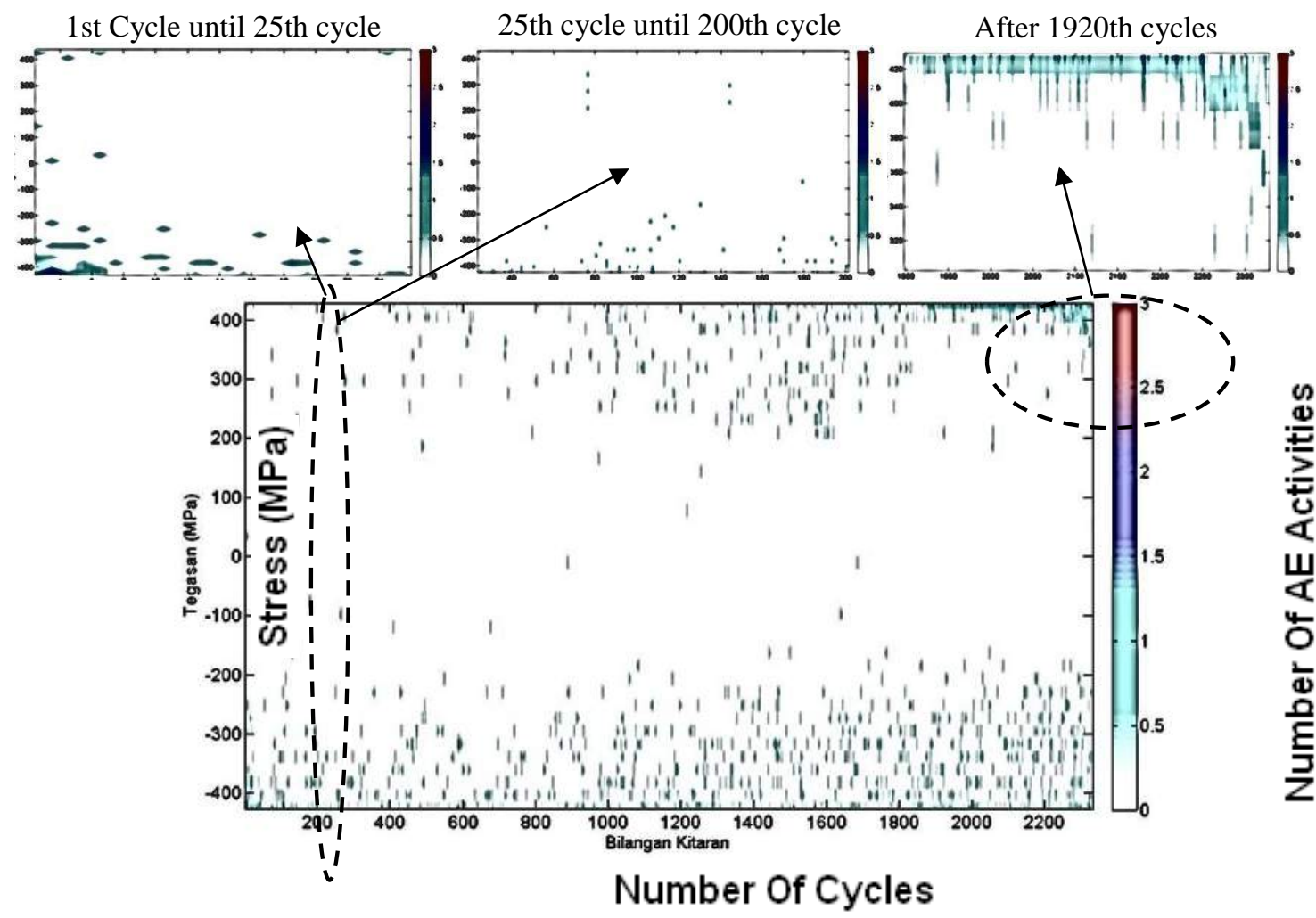

Figure 4. Acoustic emission activities during API 5L X70 fatigue test 1 with stress amplitude of $437.5 \mathrm{MPa}$.

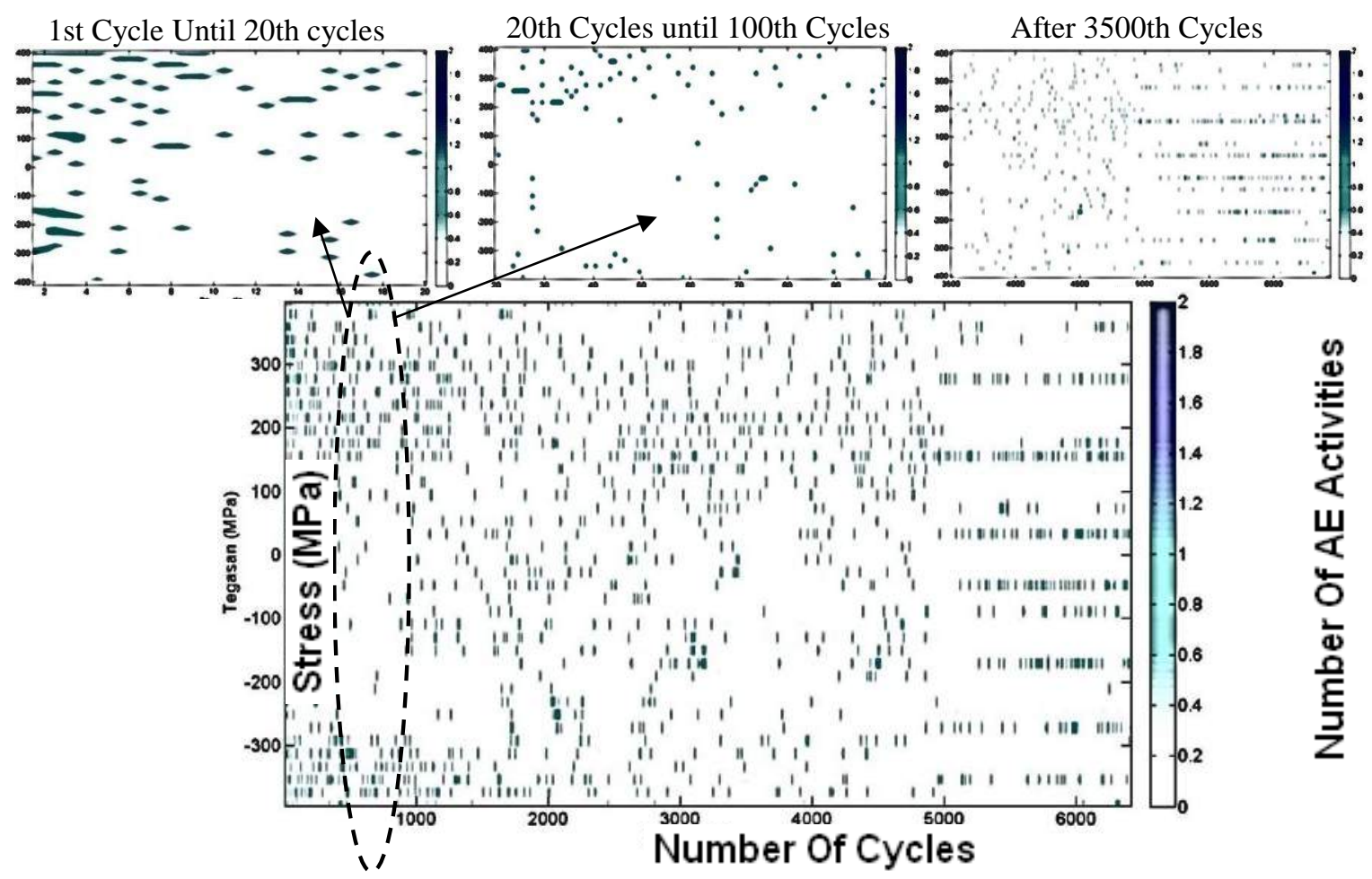

Figure 5. Acoustic emission activities during API 5L X70 fatigue test 2 with stress amplitude of $406.25 \mathrm{MPa}$. 
The AE activity shows an active behaviour from the first cycle until the 8th cycle in Figure 6. From the 8th cycle until the 1000th cycle AE activities becomes inactive with only a small number of $\mathrm{AE}$ activities per cycle found within this range. Starting at the 29379th cycle, a different phenomenon from previous tests was found. At this stage, all captured AE activities were clearly divided into three divisions. The first division shows a high number of $\mathrm{AE}$ activities within stress amplitude between $390 \mathrm{MPa}$ until $391 \mathrm{MPa}$. The second division shows active AE activities around 114.2 $\mathrm{MPa}$ until 115.5 MPa and the third division shows active AE activities around a stress amplitude of $-318 \mathrm{MPa}$ until $-315.5 \mathrm{MPa}$. AE activities from the fatigue test with stress amplitude of $53 \%$ of ultimate tensile strength are shown in Figure 7 . It is found that $\mathrm{AE}$ activities were active starting from the first cycle until the 20th cycle. AE activities become inactive until the 47088th cycle with only a small number of AE activities captured at this stage. Starting from the 47088th cycle until the 66057th cycle, $\mathrm{AE}$ activities start to become active again. The number of captured AE activities per cycle increased in every cycle during this stage. At the final stage, a phenomenon occurred similar to that which is presented in Figure 6.

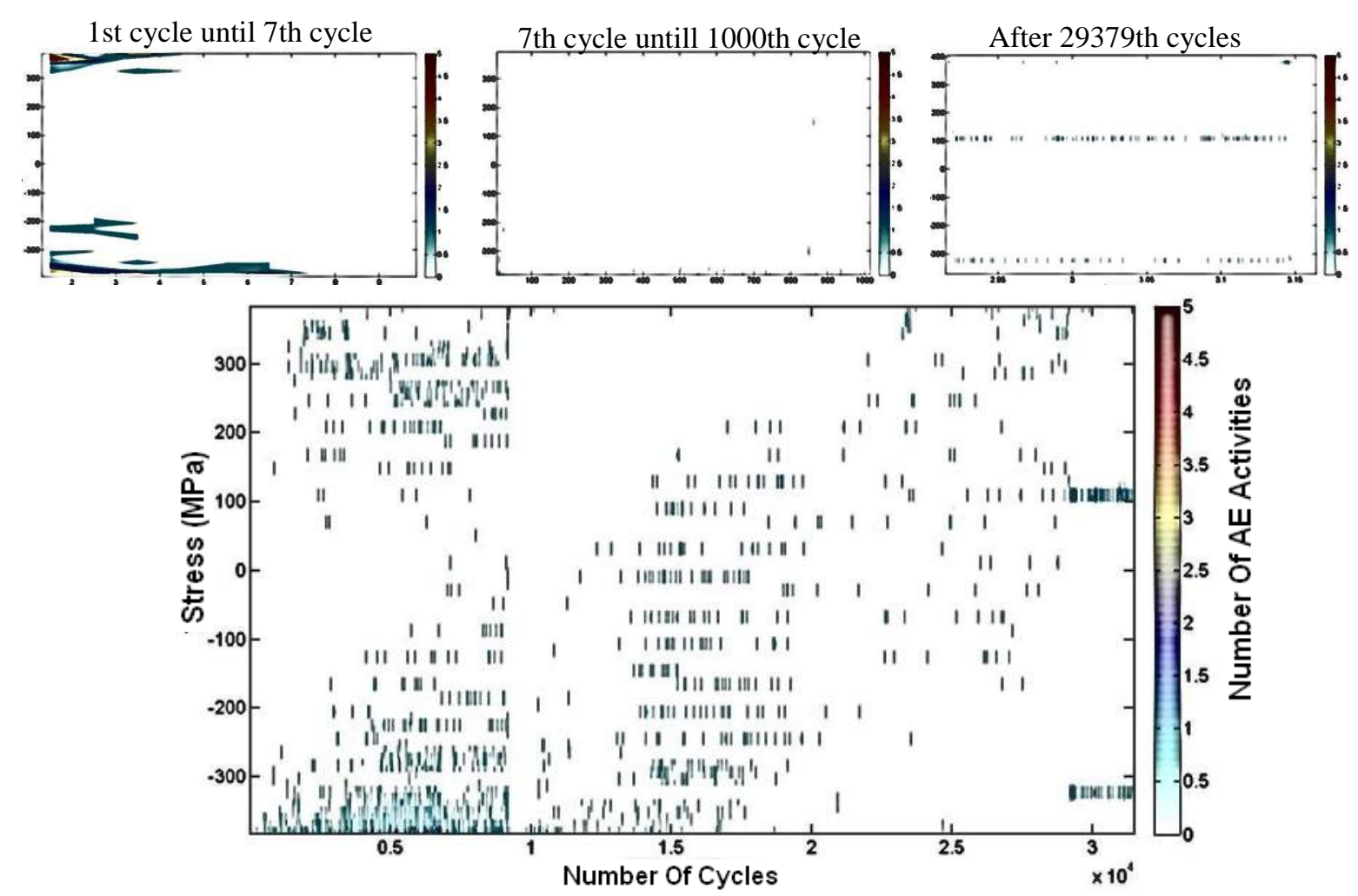

Figure 6. Acoustic emission activities during API 5L X70 fatigue test 3 with stress amplitude of 390.6 MPa.

Prior to crack initiation, all tests show high AE activities in both tension and compression modes. From Figure 8, it is found that the cyclic softening phenomenon happened at this stage. This happened because the value of cyclic peak strain amplitude started to increase in every cycle during this stage. Therefore, AE were generated due to the slip plane and microplastic deformation before the crack nucleated. AE activity at compressive peak stress is due to the Bauschinger effect, which is the phenomenon where AE are generated due to a change of strain direction (Berkovits \& Fang, 1995). It 
is presented that at the final stage, there is clear separation of $\mathrm{AE}$ activities detected at stress around zero during fatigue tests 3 and 4. The detection of AE activities around zero stress is due to crack closure phenomena where the upper crack surface contacts the lower crack surface during unloading. The phenomena of crack opening or closure was also found by Chang et al. (2009) and Berkovits \& Fang (1995), where it was detected at stress or loading around zero.
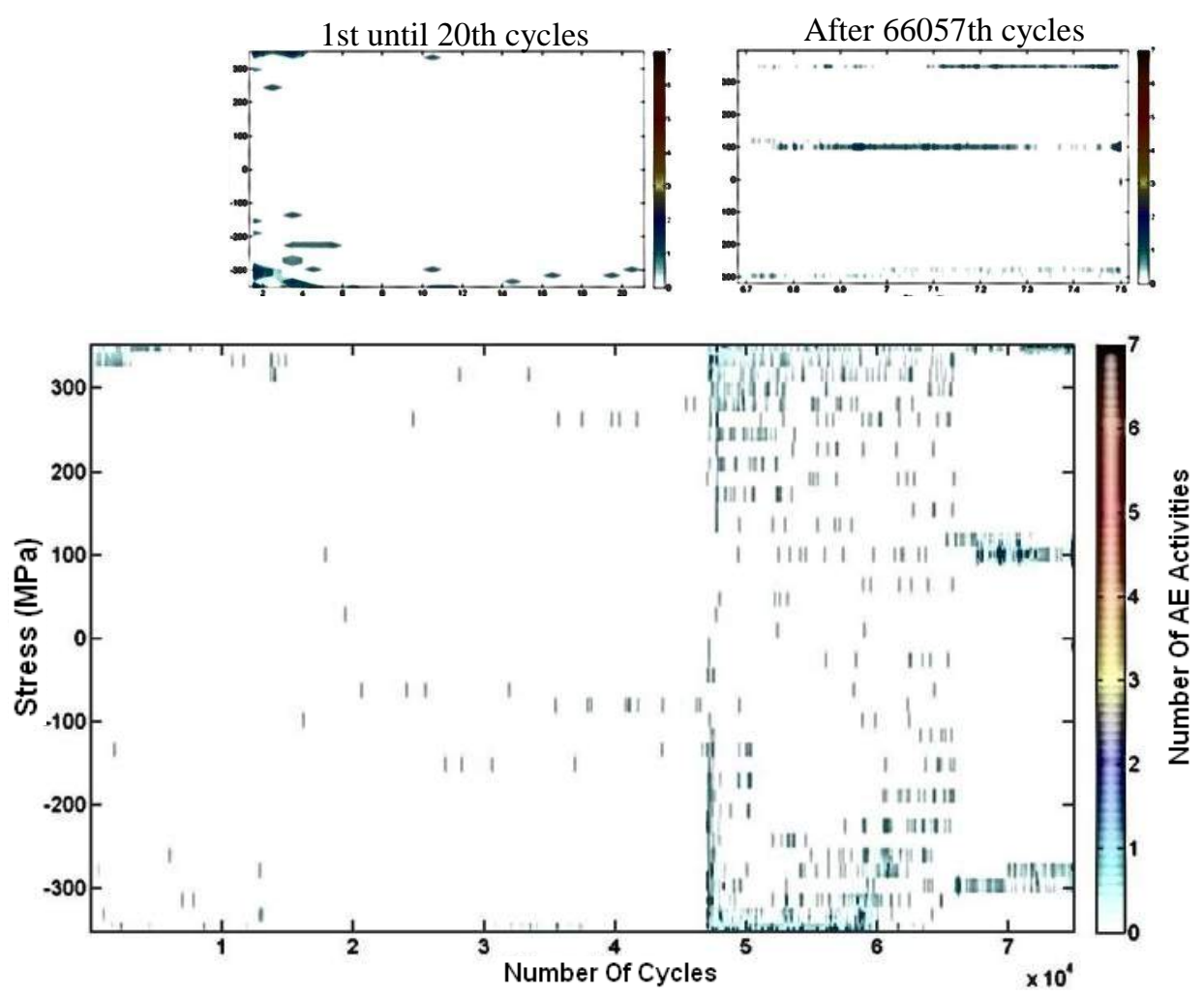

Figure 7. Acoustic emission activities during API 5L X70 fatigue test 4 with stress amplitude of 359.4MPa.

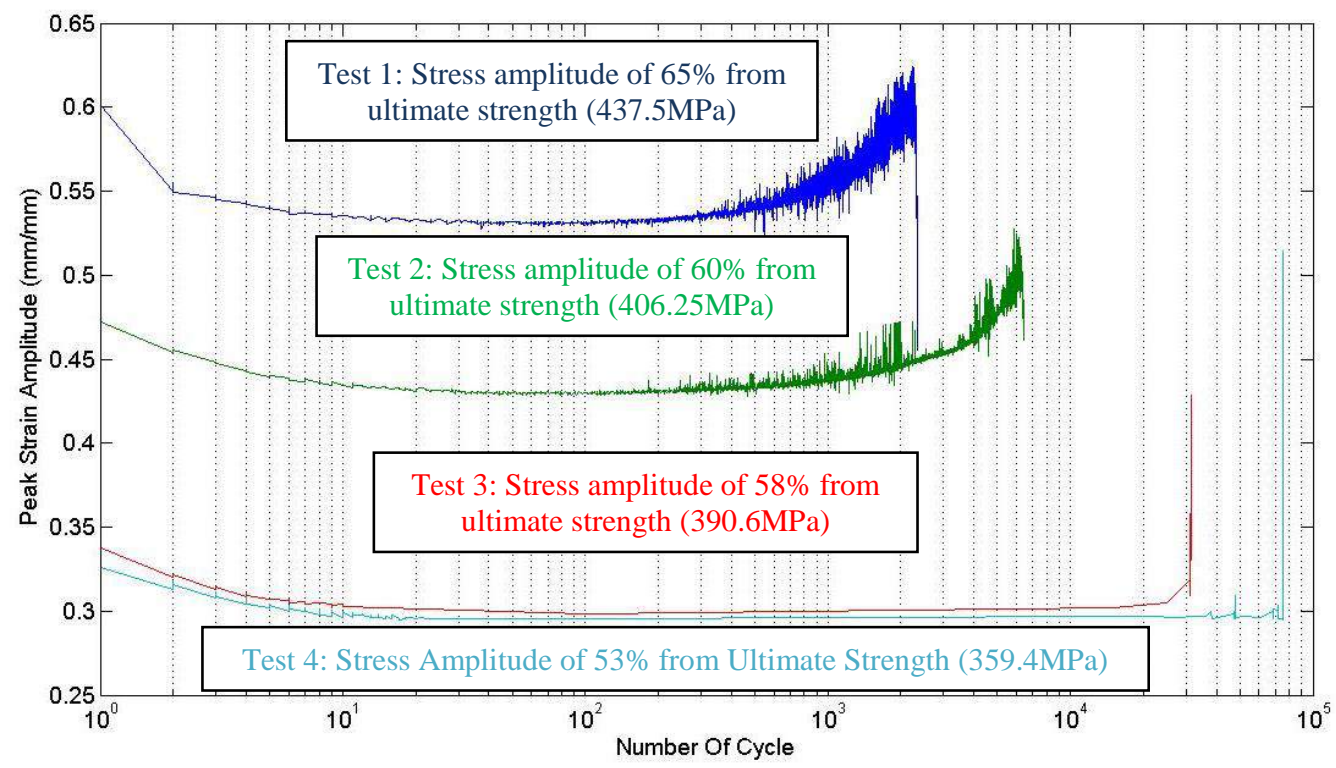

Figure 8. Cyclic peak strain amplitudes response during fatigue test. 
The fatigue mechanism can be divided into three different stages. During the first stage, cyclic softening occurred during the first cycle, which was then followed by cyclic hardening for several cycles. AE activities reactivate but the number of captured $\mathrm{AE}$ activities per cycle decreases during cyclic hardening. In the second stage, the peak strain amplitude is shown with a nearly flat response and there is no obvious change in strain during this time. This stage is called the crack incubation stage where only a small number of $\mathrm{AE}$ were captured because of pile-up breakage and formation of micro voids (Kalyanasundaram et al., 2007). However, the cyclic softening phenomenon starts at the end of the second stage and high numbers of $\mathrm{AE}$ activities are captured during this time. Most of the captured AE signatures are from slip plane formation because there is an obvious change in strain amplitude, which indicates small plastic deformation phenomena. During the last stage (the crack formation and propagation stage), the AE signatures are captured from crack initiation and propagation, crack closure and compressed crack phenomena. It is found that a higher value of stress amplitude gives a higher value of peak strain amplitude response and a longer duration of the crack incubation stage. This phenomenon shows good agreement with Chan (2009), where the crack incubation stage decreases with an increase of the local strain. The increase in cyclic stress amplitude increases the amount of local stain.

\section{Time Domain AE Signatures Behaviour}

The AE activities from all fatigue tests were divided into three different stages. At the first stage in the first few cycles, there were high AE activities due to cyclic softening. This phenomenon was caused by the start-stop effect, which affects low cycle fatigue failure (Zhong, Shan, Xiao, \& Yang, 2005). However, the start-stop effect is considered insignificant because this effect does not happen in a normal operating gas pipeline and therefore, it was decided to neglect the associated time domain AE signatures. The time domain $\mathrm{AE}$ signatures from the stage of crack incubation, microplastic deformation and slip plane formation, crack propagation and in-operation gas pipeline were selected for analysis. AE signatures from fatigue test 4 with stress amplitude of $53 \%$ of ultimate tensile strength were selected for analysis due to the obvious boundary that separates the different stages in fatigue mechanism in this case. The time domain AE signatures from different stages and different modes (tension or compression) are shown in Figure 9. One group for the time domain AE signatures from the in-operation gas pipeline is labelled as AEFM. Twenty AE signatures with similar appearance were selected from each group for analysis. The behaviour of the time domain AE signatures generated from fatigue test 4 is shown in Figure 10. It is clear that the AE signatures generated from different stages in the fatigue mechanism are burst-type AE signals. Figure 10(a and b) shows time domain AE signatures of the AE2T and AE2C group, respectively. Both groups correspond to the second stage of the fatigue mechanism in tension mode and compression mode. It is observed that time domain AE signatures from the AE2T group have a short decay time compared with that of the AE2C group signatures. The time domain AE signatures from both groups show the lowest amplitude among the time domain AE signatures from the other groups. In another observation, the AEPT group signatures in Figure 10(c) show the longest duration among all the other group time domain AE signatures with longest the rise and decay time. The shapes of the signals are quite similar with the AE2T group time domain signatures but they have different values of amplitude. In Figure 10(d), the AEPB group time domain signatures show a similar shape to that of the AE2C group time domain signatures. Although, a 
comparison between the AEPT and AE2T group time domain signatures, shows that the AEPB group time domain signatures have higher amplitudes, compared with the AE2C group time domain signatures.

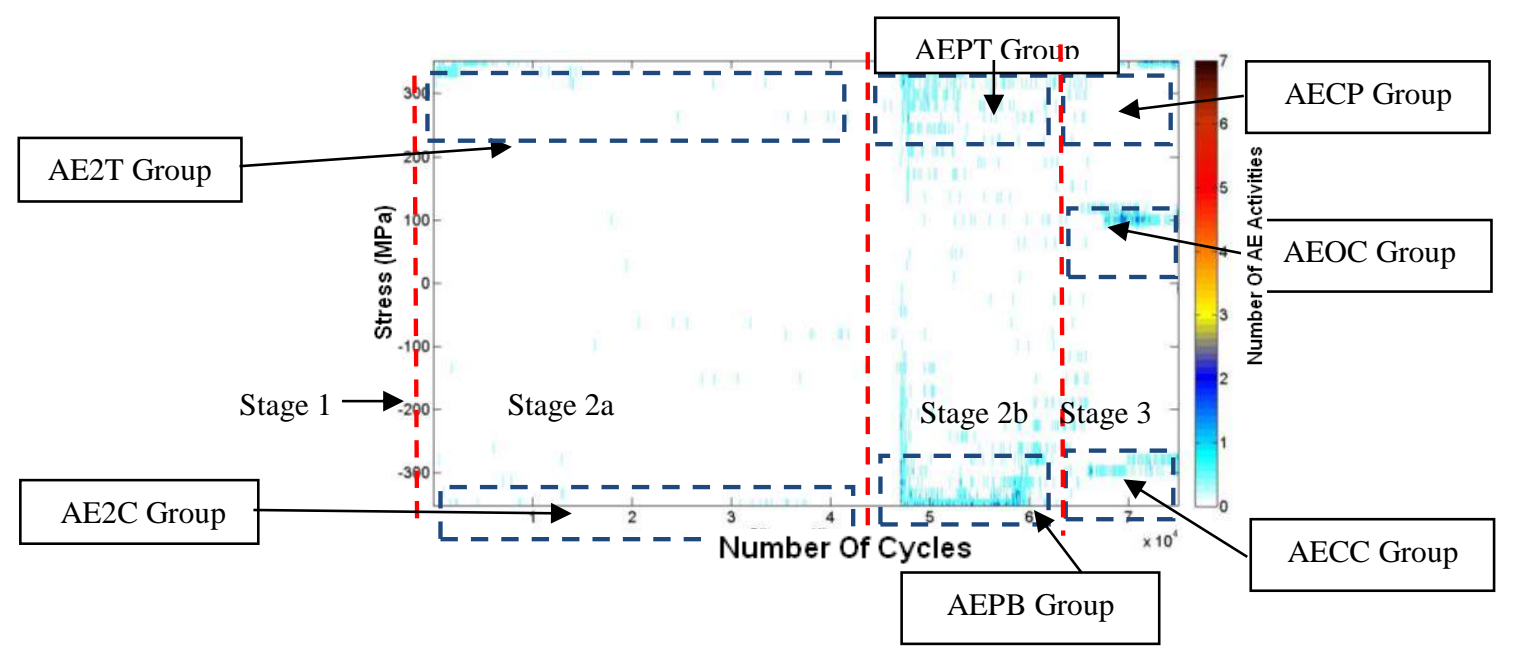

Figure 9. Group separation of micro phenomena during fatigue mechanism of API 5LX70 (Specimen 4).

The time domain $\mathrm{AE}$ signatures from the $\mathrm{AE} 2 \mathrm{~T}$ and $\mathrm{AE} 2 \mathrm{C}$ groups correspond to the time domain signatures from the crack incubation stage and show the lowest amplitudes compared with the other group's time domain AE signatures. The time domain AE signatures from the AE2T group shows the shortest duration. It has been stated that during the crack incubation stage, there is a steady-state dislocation because the AE activity is nearly quiet (Kalyanasundaram et al., 2007); only AE signatures from pile dislocation breakage and micro void formation are recorded. Janssen et al. (2004) revealed that the formation of micro voids initiates from the phenomenon of microplastic deformation in a single grain followed by the pile-up dislocation phenomenon in a stress concentration at the grain boundary. At this point, any increase in stress breaks the pile-up and then micro voids occur between the grains.

Baranov et al. (2007) found that annihilation of dislocation of a size $10^{-8}$ to $10^{-5}$ $\mathrm{m}$ long produces time domain AE signatures with small amplitudes and durations of $5 \times$ $10^{-5} \mu \mathrm{s}$. The term annihilation of dislocation refers to the dislocation breakage. Therefore, for the AE2T group, the source of the time domain AE signatures is the pileup dislocation breakage. It is found that there is a very small plastic deformation that occurs prior to crack formation. The time domain AE signatures from the AE2T and AEPT groups show the difference in amplitudes and duration, due to the Bauschinger effect, occur at compressive peak stress (Berkovits \& Fang, 1995). The similar shape of the time domain AE signatures from the AE2C and AEPB groups are due to the Bauschinger effect. The time domain AE signatures from the AECP, AEOC and AECC groups are shown in Figure 10(e-g), respectively. In the crack stage, all time domain signatures show a short burst behaviour with very short rise time. In Figure 10(e), the time domain $\mathrm{AE}$ signatures from the $\mathrm{AECP}$ group produces a very high amplitude signal followed by the AEOC group in Figure 10(f) with a similar shape. In Figure $10(\mathrm{~g})$, the time domain AE signatures from the AECC group show the lowest amplitude with a nearly similar shape to the AE2C group. During crack initiation, burst-type AE appeared with very high amplitude and energy (Ai et al., 2010; Maslov \& Gradov, 
1986), while the AE burst from the crack closure phenomenon has lower amplitude (Lee, Rhyim, Kwon, \& Ono, 1995; Chang et al., 2007). AE signatures from the AECP and AEOC groups have similar burst behaviour and shape but different amplitudes. Figure 10(h) shows the time domain AE signatures captured from the normal operating condition of a gas pipeline during field measurements. It shows the very high-amplitude burst-type signal compared with the AE signatures from the AECP group. AE signatures from the normal operating condition of a gas pipeline show a smooth pattern with less complexity.

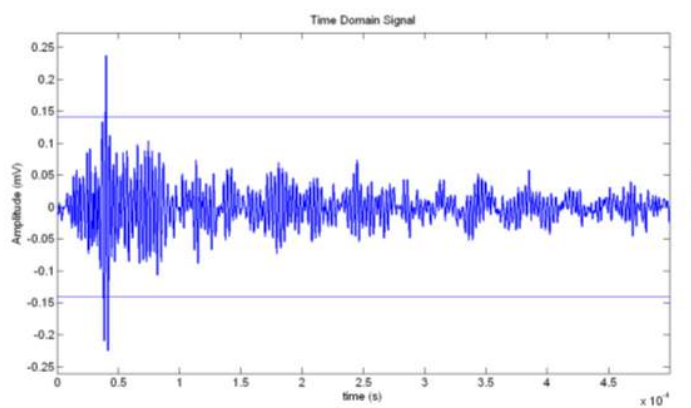

(a) AE2T

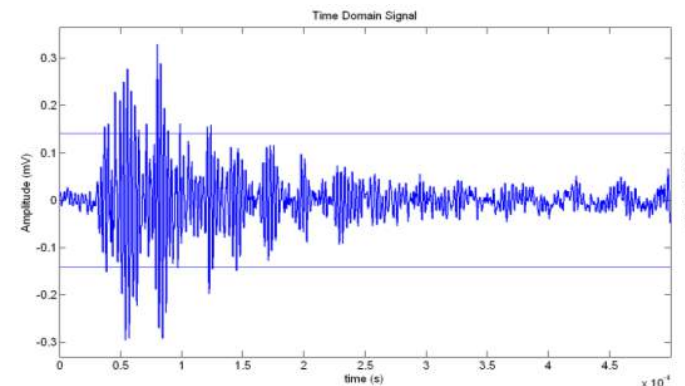

(c) AEPT

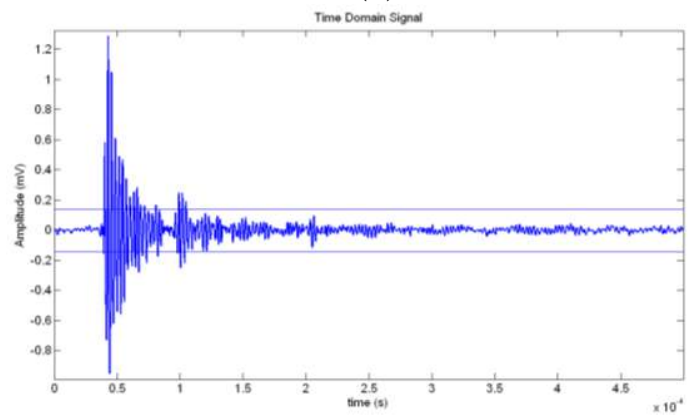

(e) AECP

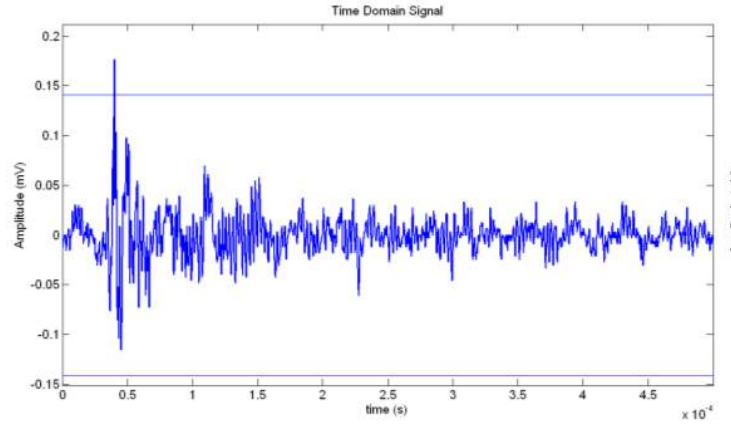

(g) AECC

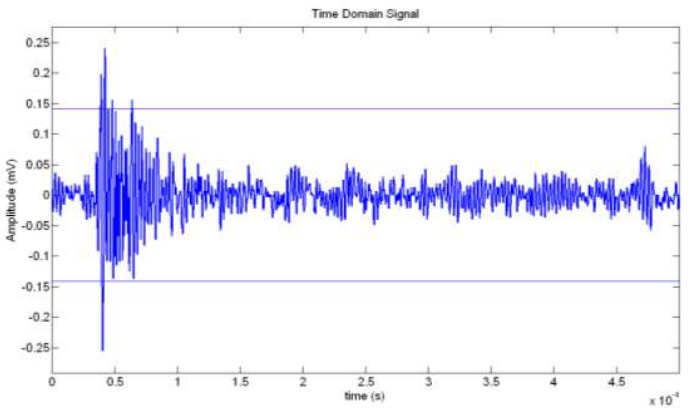

(b) $\mathrm{AE} 2 \mathrm{C}$

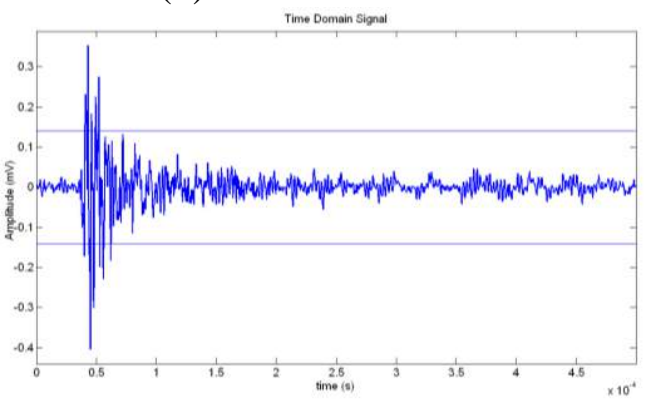

(d) AEPB

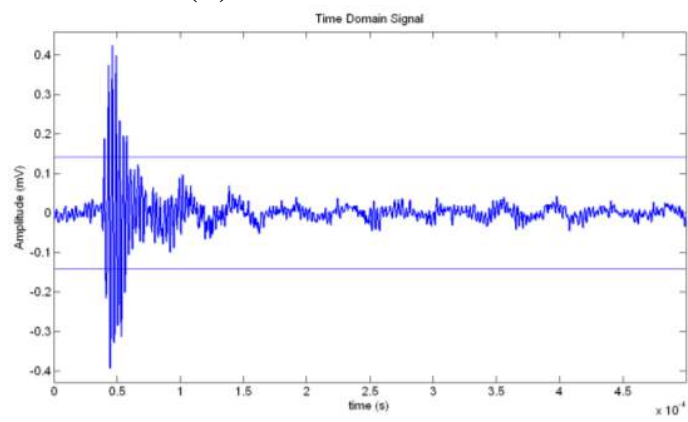

(f) $\mathrm{AEOC}$

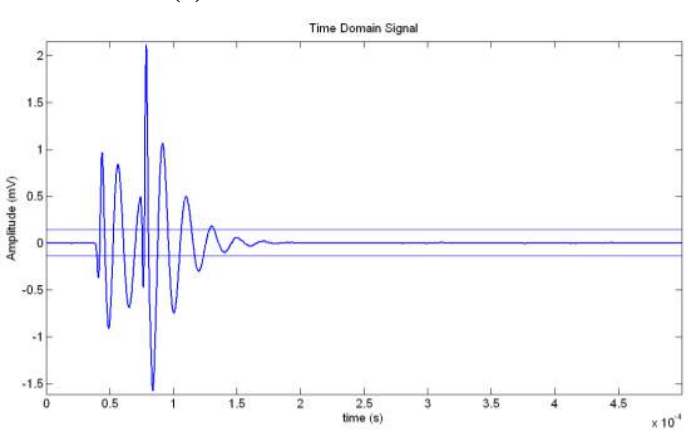

(h) AEFM

Figure 10. Acoustic emission signatures during test 1 fatigue and field measurement. 


\section{Feature Extraction}

AE maximum amplitude and energy extracted from the time domain signatures are presented in Table 1. The mean value of AE maximum amplitude from the AE2T group is $47.9 \mathrm{~dB}$, while the value is $46.08 \mathrm{~dB}$ for the AE2C group. During the stage of microplastic deformation and slip plane formation, the mean value of $\mathrm{AE}$ maximum amplitude increases. The crack initiation and propagation from the AECP group gives a mean value of $\mathrm{AE}$ maximum amplitude of $63.37 \mathrm{~dB}$. The maximum amplitude value for the AEFM group shows the highest mean value of $64.84 \mathrm{~dB}$. The mean of AE energy for both the fatigue mechanism and in-operation gas pipeline show a similar pattern with mean of maximum amplitude. In the crack incubation stage, the mean of $\mathrm{AE}$ energy from the AE2T group is $31.52 \mathrm{eu}$, while the value drops to 30.82 eu for the AE2C group. At the stage of microplastic deformation and slip plane formation, the mean of AE energy from the AEPT and AEPB groups increases. The interesting finding was that the mean of AE energy from the normal operating gas pipeline is far higher than during the crack propagation stage with a value of $2522 \mathrm{eu}$.

Table 1. Range and mean value of AE maximum amplitude and energy.

\begin{tabular}{ccccc}
\hline Signal & $\begin{array}{c}\text { Maximum } \\
\text { Amplitude } \\
(\mathrm{dB})\end{array}$ & $\begin{array}{c}\text { Mean of } \\
\text { Maximum } \\
\text { Amplitude } \\
(\mathrm{dB})\end{array}$ & Energy $(\mathrm{eu})$ & $\begin{array}{c}\text { Mean of } \\
\text { Energy } \\
(\mathrm{eu})\end{array}$ \\
\hline AE2T & $47.1-48.6$ & 47.91 & $20.9-45.3$ & 31.52 \\
AE2C & $44.1-48.3$ & 46.08 & $17.0-45.2$ & 30.82 \\
AEPT & $50.1-50.9$ & 50.38 & $125-182$ & 156.7 \\
AEPB & $50.1-62.6$ & 53.74 & $46.4-278$ & 178.56 \\
AECP & $61.4-64.8$ & 63.37 & $481-1210$ & 778.2 \\
AEOC & $52.4-57.7$ & 54.29 & $75-293$ & 117.72 \\
AECC & $44.5-48.3$ & 46.02 & $9.59-39$ & 20.53 \\
AEFM & $60.3-68.2$ & 64.84 & $1070-3820$ & 2522 \\
\hline
\end{tabular}

The mean of AE maximum amplitude and energy from the in-operation gas pipeline was higher than the values from the fatigue mechanism. When neglecting the last stage of fatigue mechanism, the $\mathrm{AE}$ maximum amplitude and energy show an obvious difference compared with the in-operation gas pipeline. However, detection of symptoms of a fault below the lower value of normal operating conditions is not essential. This is because the lower maximum amplitude and energy values are interpreted as noise. This shows that the AE maximum amplitude and energy are sufficiently sensitive to differentiate three different stages of crack mechanism but are unsuitable to differentiate between the normal and fatigue conditions of a gas pipeline.

The range and mean value of selected statistical parameters are shown in Table 2. All statistical parameters were obtained from the time AE signatures. As observed during the crack incubation stage, the mean value of standard deviation from the AE2T and AE2C groups are 0.03011 and 0.02667 , respectively. These values increase to 0.0566 for the AEPT group and to 0.0568 for the AEPB group during microplastic deformation and slip plane formation. During crack propagation, the mean of the standard deviations of the AECP group increased. When the crack opening and 
closure phenomena exist, the standard deviation from the AEOC group was dropped. The standard deviation from the AECC group is the lowest value and corresponds to the compressed crack. AE maximum amplitude and energy and the mean of the standard deviations from the normal operating gas pipeline are higher than those of the fatigue mechanism. During crack propagation and crack opening and closure, the AECP and AEOC groups show high kurtosis. The trend of standard deviation mean values is similar to the trend of the AE maximum amplitude and energy. Therefore, it can be concluded that the standard deviation values are able to differentiate the different stages of the fatigue mechanism. In contrast, the trend of kurtosis of the time domain $\mathrm{AE}$ signatures from the fatigue mechanism does not show any obvious difference. Nevertheless, it could separate between the in-operation gas pipeline and the crack stage, corresponding to the AECP and AEOC groups. It can be observed that the skewness value is directly related to loading direction. During tension modes of cyclic stress, the skewness of the AE2T, AEPT, AECP and AEOC groups give positive values, whereas the AE2C, AEPB and AECC groups give negative values. This indicates that the normal operating condition load acts on tension due to the positive value of skewness of the AEFM group. It is found that like kurtosis, the skewness can also detect the crack stage when the fatigue mechanism is in tensile mode.

Table 2. Range and mean value of standard deviation, kurtosis and skewness.

\begin{tabular}{ccccccc}
\hline $\begin{array}{c}\text { Signal } \\
\text { Group }\end{array}$ & $\begin{array}{c}\text { Standard } \\
\text { Deviation }\end{array}$ & $\begin{array}{c}\text { Mean of } \\
\text { Standard } \\
\text { Deviation }\end{array}$ & Kurtosis & $\begin{array}{c}\text { Mean of } \\
\text { Kurtosis }\end{array}$ & Skewness & $\begin{array}{c}\text { Mean of } \\
\text { Skewness }\end{array}$ \\
\hline AE2T & $0.0264-0.03425$ & 0.0301 & $9.8-30.15$ & 22.77 & $-0.623-0.664$ & 0.0082 \\
AE2C & $0.0212-0.0351$ & 0.0267 & $7.71-30.32$ & 12.92 & $-0.45-0.538$ & -0.056 \\
AEPT & $0.0528-0.0602$ & 0.0566 & $9.22-11.34$ & 10 & $-0.236-0.374$ & 0.065 \\
AEPB & $0.0315-0.1078$ & 0.0568 & $6.53-48.92$ & 21.75 & $-1.462-0.103$ & -0.286 \\
AECP & $0.098-0.1551$ & 0.1237 & $38.35-49.35$ & 45.75 & $0.82-1.467$ & 1.132 \\
AEOC & $0.0392-0.077$ & 0.048 & $23.8-63.37$ & 44.41 & $-0.581-1.02$ & 0.34 \\
AECC & $0.017-0.0282$ & 0.022 & $9.12-29.44$ & 17.55 & $-1.046-0.996$ & -0.105 \\
AEFM & $0.177-0.276$ & 0.228 & $10.48-33.13$ & 20.64 & $-0.713-2.446$ & 0.774 \\
\hline
\end{tabular}

\section{CONCLUSIONS}

Monitoring and assessment of the time domain AE signatures during fatigue mechanism and in-operation gas pipeline has been carried out. The distribution of AE counts during the fatigue mechanism can be divided into three different stages: crack incubation stage; microplastic deformation and slip plane formation stage; and crack formation and propagation stage. The larger amplitude loading shortens the crack incubation stage. Crack initiations are indicated by rapid increases of AE activities at positive peak stress. $\mathrm{AE}$ activities around zero stress correspond to crack closure phenomena. AE maximum amplitude and energy from the normal operating pipeline show higher values than for crack propagation. This phenomenon is not suitable for condition monitoring purposes. Analysis of the kurtosis can separate signatures from the normal operating pipeline and crack propagation and closure. Skewness is a good parameter for separating signatures from different load directions. However, it is not suitable to differentiate signatures between the normal operating pipeline and the fatigue mechanism. The dominant frequencies of $\mathrm{AE}$ are found to separate signatures from the fatigue mechanism and the 
normal operating pipeline. Kurtosis is also promising for detecting micro phenomena during the crack stage.

\section{REFERENCES}

Ai, Q., Liu, C. X., Chen, X. R., He, P., \& Wang, Y. (2010). Acoustic emission of fatigue crack in pressure pipe under cyclic pressure. Nuclear Engineering and Design, 240, 3616-3620.

Baranov, V., Kudryavtsev, E., Sarychev, G., \& Shcavelin, V. (2007). Acoustic emission in friction. First ed. United Kingdom: Elsevier.

Berkovits, A., \& Fang, D. (1995). Study of fatigue crack characteristic by acoustic emission. Engineering Fracture Mechanics, 51(3), 401-406.

Chan, K. S. (2009). Roles of microstructure in fatigue crack initiation. International Journal of Fatigue, 32(9), 1428-1447.

Chang, H., Han, E. H., Wang, J. Q., \& Ke, W. (2009). Acoustic emission study of fatigue crack closure of physical short and long cracks for aluminum alloy LY12CZ. International Journal of Fatigue, 31, 403-407.

Elangovan, M., BabuDevasenapati, S., Sakhtivel, N. R., \& Ramachandran, K. I. (2011). Evaluation of expert system for condition monitoring of a single point cutting tool using principle component analysis and decision tree algorithm. Expert System with Application, 38, 4450-4459.

Huang, M., Jiang, L., Liaw, P. K., Brooks, C. R., Seelay, R., \& Klarstrom, D. L. (1998). Using acoustic emission in fatigue and fracture materials research. JOMe, 50(11):1. Retrieved from http://www.tms.org/pubs/journals/JOM/9811/Huang/ Huang-9811.html

Janssen, M., Zuidema, J., \& Wanhill, R. J. H. (2004). Fracture mechanic. 2nd Edition. London: Spon Press.

Kalyanasundaram, P., Mudhophadyay, C. K., \& SubraRao, S. V. (2007). Practical acoustic emission. First ed. United Kingdom: Alpha Science International Ltd.

Lee, C. S., Rhyim, Y. M., Kwon, D., \& Ono, K. (1995). Acoustic emission measurement of fatigue crack closure, ScriptaMetallurgicaet. Materialia, 32(5), 701-706.

Maslov, L. I., \& Gradov, O. M. (1986). Fracture energy analysis via acoustic emission. International Journal of Fatigue, 8(2), 67-71.

Muhlbauer, W. K. (2004). Pipeline risk management: ideas, techniques and resources. Third ed. United States: Gulf Professional Publishing.

Nuawi, M. Z., Abdullah, S., Abdullah, S., Haris, S. M., \& Arifin, A. K. (2009). MATLAB: A Comprehensive reference for engineers. First Edition, Malaysia: McGraw Hill.

Roberts, T. M., \& Talebzadeh, M. (2003). Acoustic emission monitoring of fatigue crack prkopagation. Journal of Constructional Steel Research, 59, 695-712.

Zhong, Y., Shan, Y., Xiao, F., \& Yang, K. (2005). Effect of toughness on low cycle fatigue behavior of pipeline steels. Material Letters, 59(14-15), 1780-1784. 